Diabetologe 2021 · 17:241-242

https://doi.org/10.1007/s11428-021-00751-x

Angenommen: 5. März 2021

(c) Springer Medizin Verlag $\mathrm{GmbH}$, ein Teil von Springer Nature 2021

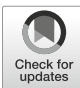

Matthias Kaltheuner ${ }^{1}$. Lutz Heinemann ${ }^{2}$

'Diabetologikum Leverkusen, Leverkusen, Deutschland

${ }^{2}$ Science Consulting in Diabetes $\mathrm{GmbH}$, Neuss, Deutschland

\title{
Einsatz digitaler Tools in der Behandlung von Patienten mit Diabetes
}

\section{Die Welt dreht sich immer schneller!}

Wenn die diabetologische Behandlung von Patienten mit Diabetes - unabhängig davon in welchem Umfeld - heutzutage überwiegend aus dem Erfassen, Dokumentieren, Analysieren und Interpretieren von Daten besteht, ist es folgerichtig, die ärztlich-menschlichen Fähigkeiten und den Einsatz von Informationstechnologie (IT) entsprechend auszurichten. Dies geschieht vor dem Hintergrund der massiven Nutzung digitaler Technologien in unserem Gesamtumfeld und im Gesundheitswesen, aktuell zusätzlich angetrieben von einer Pandemie. Ziel der Beiträge in diesem Heft ist es, die verschiedenen Facetten dieser Entwicklungen darzustellen, wobei sich in der Behandlung der Patienten in den letzten Jahren vieles ereignet und geändert hat, wir befinden uns offensichtlich mitten in einem weitreichenden Transformationsprozess. Wir haben unsere Kollegen gebeten, über die aktuellen Entwicklungen in verschiedenen Bereichen zu berichten, auch mit einem Blick in die Zukunft. Dabei ist dieser natürlich sehr unsicher, so setzen sich viele Dinge, von denen wir vor 10 Jahren gedacht hatten, sie würden eintreten, nicht durch, dafür andere, die wir nicht als relevant eingeschätzt hatten.

Eindeutig ist, Diabetologie ist in vielerlei Hinsicht primär Informationsverarbeitung, deshalb liegt der Fokus verschiedener Beiträge auch hierauf. Daraus ergibt sich, dass die medizinischen Einrichtungen ihre Prozessgestaltung den neuen digitalen Tools anpassen müssen, d.h. IT darf kein Fremdkörper bei der Arbeit darstellen, um den sich ein Ex- perte kümmern muss. Wenn Datenverarbeitung ein zentraler Aspekt der alltäglichen ärztlichen Arbeit ist, ist es schwer vorstellbar, dass ein externer Experte die Transformation der Arbeitsprozesse alleine sachgerecht erledigen kann. Insofern erscheint uns die Kompetenz von Ärzten über ihre Informationsverarbeitung und deren Transformation in digitale Prozesse als ausgesprochen wichtig. In 2 Beiträgen wird nicht nur beleuchtet, wo die ambulante und die stationäre Diabetologie diesbezüglich aktuell einzuordnen sind („state of the art“), sondern es werden auch sehr konkrete Hinweise und Beispiele gegeben, wie die Entwicklung der digitalen Prozesse in der Patientenversorgung verlaufen wird.

\section{》) Informationstechnologie darf keinen Fremdkörper bei der praktischen Arbeit darstellen}

Dass Deutschland beim Einsatz digitaler Tools in der Betreuung von Patienten mit Diabetes sowohl im europäischen als auch im internationalen Vergleich recht gut positioniert ist, ist auch den Aktivitäten der zuständigen Fachgesellschaft geschuldet. Deren Beitrag zu den nationalen Großprojekten wie der ePA (elektronische Patientenakte) und damit auch der eDA (elektronische Diabetesakte) wird hoffentlich essenziell dazu beitragen, Informationsübermittlungsdefizite zu überwinden.

Ein enorm wichtiger und kritischer Aspekt ist die Frage: Nützen die digita- len Tools konkret den Betroffenen bei der Bewältigung ihrer Erkrankung oder erfahren diese dadurch eine weitere Belastung? Ein ausgewiesener Fachmann nimmt Stellung dazu, ob solche Tools dabei helfen, emotionale Belastungen abzubauen. Dabei stehen subjektive Ziele neben objektiven.

Die Menschen, die hinter der DiaDigital-Gruppe stehen, haben durch die intensive Beschäftigung mit Apps viel an Kompetenz in diesem speziellen Bereich entwickelt, welches auch deren Entwicklern zugutekommt. Dabei wurde auch ersichtlich: Es wird nur das genutzt, was nützt. Damit die Entwicklung in Deutschland nicht nur von den Herstellern entsprechender Produkte vorangetrieben wird, bedarf es einer aktiven Fachgesellschaft (s. oben), unterstützt von entsprechenden Arbeitsgemeinschaften. Da die Gesundheitspolitik die Nutzung digitaler Tools in einem Ausmaß unterstützt und vorantreibt, welches vor kurzem noch undenkbar war, verläuft deren Entwicklung jetzt erheblich beschleunigt.

Uns ist es wichtig, dass bei der Behandlung der Betroffenen das ärztliche Handeln nicht zu kurz kommt und Patienten und Ärzte eine Chance haben, die digitale Transformation konkret mitzugestalten. Die ärztliche Gesprächsführung bleibt weiterhin ein wichtiger Informationsprozess, ob sie nun im persönlichen Kontakt, per Telefon oder Videokonferenz erfolgt. Insgesamt soll ein sinnvoller und sicherer Einsatz digitaler Tools dazu führen, dass die Effektivität und Effizienz 


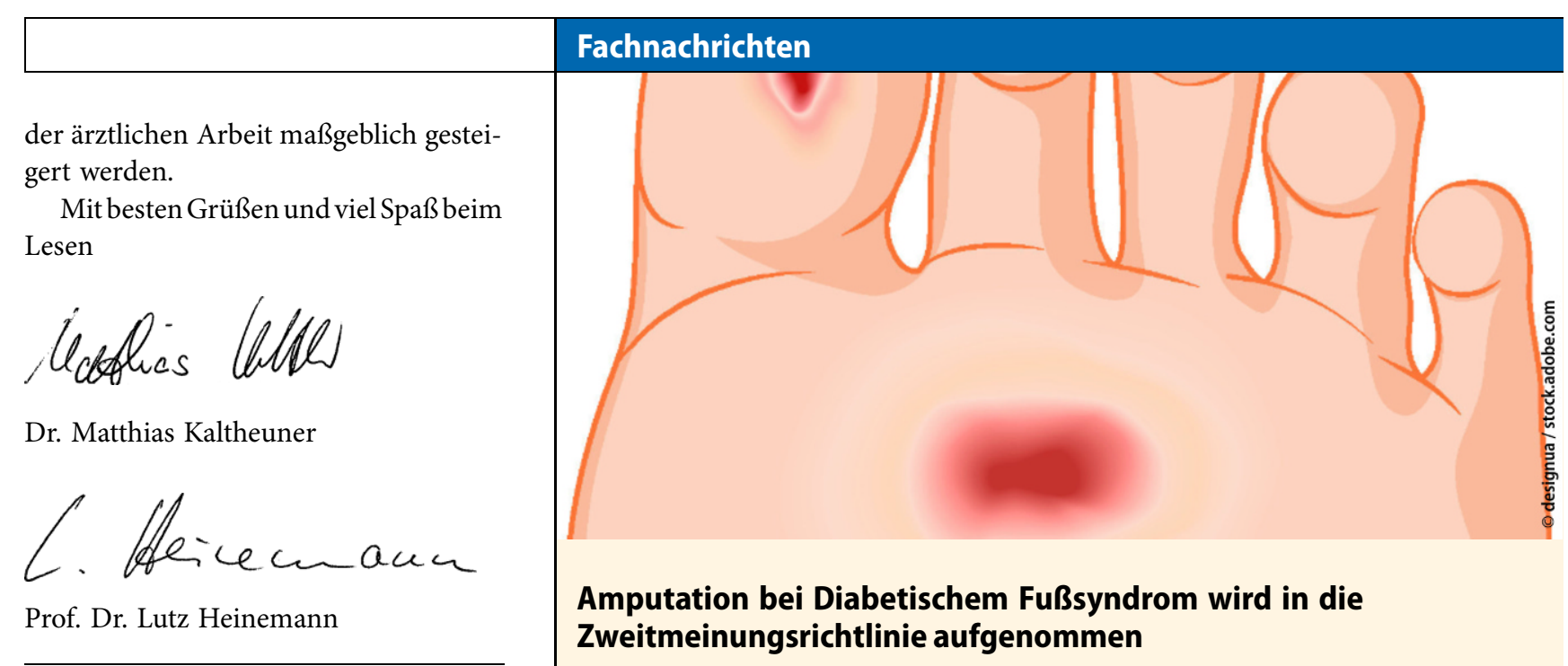

\section{Korrespondenzadresse}

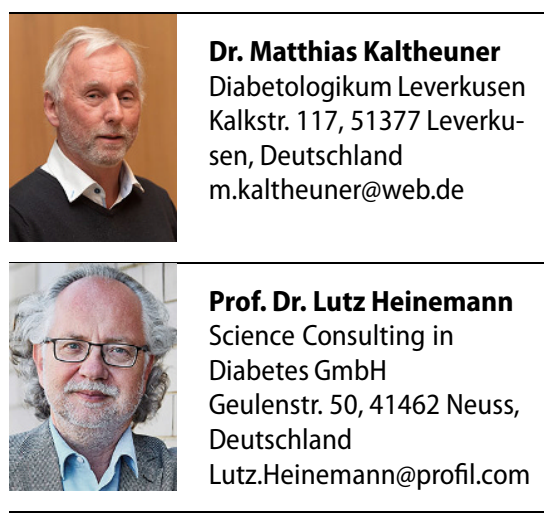

Interessenkonflikt. M. Kaltheuner und L. Heinemann geben an, dass kein Interessenkonflikt besteht.
Hierzulande werden jährlich bis zu 50.000

Amputationen aufgrund eines diabetischen Fußsyndroms (DFS) - eine der häufigsten Folgeerkrankungen bei Diabetes - vorgenommen. Die Arbeitsgemeinschaft „Diabetischer Fuß" der Deutschen Diabetes Gesellschaft (DDG) kämpft schon lange darum, dass solch schwerwiegende medizinische Entscheidungen durch eine Zweitmeinung abgesichert werden. Dazu liegt nun ein überarbeiteter Beschluss des Gemeinsamen Bundesausschusses (G-BA) dem Bundesgesundheitsministerium vor. "Wir betrachten die G-BA-Entscheidung als vollen Erfolg", erklärt Dr. med. Michael Eckhard, Vorsitzender der AG „Diabetischer Fuß". Die Arbeitsgemeinschaft setzt sich auch im Rahmen des Zertifizierungsverfahrens von Behandlungseinrichtungen dafür ein, amputationsbedrohte Extremitäten bei Menschen mit Diabetes zu erhalten. „Der Rechtsanspruch auf die Einholung einer unabhängigen ärztlichen Zweitmeinung soll Betroffene unterstützen, eine Entscheidung zur möglichen Auswahl vorgeschlagener Behandlungsmöglichkeiten zu treffen und damit gegebenenfalls eine medizinisch nicht gebotene Amputation zu vermeiden", erklärt der Diabetologe.

Einen Antrag auf Zulassung zum Zweitmeinungsverfahren zu einem möglichen Amputationserfordernis aufgrund eines DFS können grundsätzlich Fachärzt*innen aus acht Disziplinen stellen. Dazu gehören folgende Fachrichtungen: Innere Medizin und Angiologie, Innere Medizin und Endokrinologie und Diabetologie, Innere Medizin mit Zusatzbezeichnung Diabetologie, Allgemeinmedizin mit Zusatzbezeichnung Diabetologie, Gefäß- chirurgie, Orthopädie und Unfallchirurgie, Orthopädie oder Chirurgie mit Schwerpunkt Unfallchirurgie, Allgemeinchirurgie sowie Plastische, Rekonstruktive und Ästhetische Chirurgie. Außerdem wird es möglich sein, auch Angehörige nichtärztlicher Fachberufe in die Zweitmeinungsberatungen einzubeziehen, die zum multiprofessionellen Behandlungsteam von Menschen mit DFS gehören. Dazu zählen unter anderem Podologen, Orthopädieschuhmacher sowie Orthopädietechniker und Orthopädiemechaniker. Von allen Zweitmeinern wird der Nachweis einer besonderen Kompetenz gefordert, die zeigt, dass sie „für die Behandlung des diabetischen Fußsyndroms besonders qualifiziert sind, und dass deren Expertise bei Abgabe der Zweitmeinung bei Bedarf genutzt werden kann", so der Wortlaut des Beschlusses. Als besonders qualifiziert gilt laut der Zweitmeinungsrichtlinie, wer mindestens bereits fünf Jahre regelmäßig aktiv in der Versorgung von Patient*innen mit DFS tätig ist und so mindestens 30 DFSPatient*innen in einem multidisziplinären Setting behandelt. „Der künftig geforderte Nachweis dieser besonderen Expertise für das DFS ist sehr zu begrüßen, weil allein der Erwerb eines Facharztstatus noch lange nicht Beleg dafür ist, dass jemand wirklich über eine besondere Qualifikation in diesem speziellen Teilbereich verfügt", so Eckhard. Die Zweitmeinungsrichtlinie zur Amputation bei DFS muss nun noch durch das Bundesgesundheitsministerium ratifiziert werden, was als Formalie gilt.

Quelle: www.deutsche-diabetesgesellschaft.de 